\title{
A new class of non-aligned Einstein-Maxwell solutions with a geodesic, shearfree and non-expanding multiple Debever-Penrose vector
}

\author{
Norbert Van den Bergh \\ Ghent University, Department of Mathematical Analysis FEA16, \\ Galglaan 2, 9000 Ghent, Belgium \\ E-mail: norbert.vandenbergh@ugent.be
}

\begin{abstract}
In a recent study of algebraically special Einstein-Maxwell fields[1] it was shown that, for non-zero cosmological constant, non-aligned solutions cannot have a geodesic and shearfree multiple Debever-Penrose vector $\boldsymbol{k}$. When $\Lambda=0$ such solutions do exist and can be classified, after fixing the null-tetrad such that $\Psi_{0}=\Psi_{1}=\Phi_{1}=0$ and $\Phi_{0}=1$, according to whether the Newman-Penrose coefficient $\pi$ is 0 or not. The family $\pi=0$ contains the Griffiths solutions[2], with as sub-families the Cahen-Spelkens, Cahen-Leroy and Szekeres metrics. It was claimed in [2] and repeated in [1] that for $\pi=0$ both null-rays $k$ and $\ell$ are non-twisting $(\bar{\rho}-\rho=\bar{\mu}-\mu=0)$ : while it is certainly true that $\mu(\bar{\rho}-\rho)=0$, the case $\mu=0$ appears to have been overlooked. A family of solutions is presented in which $k$ is twisting but non-expanding.
\end{abstract}

PACS numbers: $04.20 . \mathrm{Jb}$

\section{Introduction}

In the quest for exact solutions of the Einstein-Maxwell equations

$$
R_{a b}-\frac{1}{2} R g_{a b}+\Lambda g_{a b}=F_{a c} F_{b}^{c}-\frac{1}{4} g_{a b} F_{c d} F^{c d},
$$

a large amount of research has been devoted to the study of aligned Einstein-Maxwell fields, in which at least one of the principal null directions (PND's) of the electromagnetic field tensor $\boldsymbol{F}$ is parallel to a PND of the Weyl tensor, a so called Debever-Penrose direction, see [6] and citations therein. A systematic attempt at classifying the algebraically special non-aligned solutions was initiated in [1]. One of the topics considered, dealing with the reverse of the Goldberg-Sachs theorem, enquired after the existence of algebraically special (non-conformally flat and non-null) Einstein-Maxwell fields with a possible nonzero cosmological constant for which the multiple Weyl-PND $\boldsymbol{k}$ is geodesic and shear-free $\neq$ $\left(\Psi_{0}=\Psi_{1}=\kappa=\sigma=0\right)$ and for which $\boldsymbol{k}$ is not parallel to a PND of $\boldsymbol{F}\left(\Phi_{0} \neq 0\right)$. In order to avoid frequent referring to the equations of [1] I present the commutator relations, GHP,

$\$$ Throughout I use the sign conventions and notations of [6] \$7.4, with the tetrad basis vectors taken as $\boldsymbol{k}, \boldsymbol{\ell}, \boldsymbol{m}, \overline{\boldsymbol{m}}$ with $-k^{a} \ell_{a}=1=m^{a} \overline{\boldsymbol{m}}_{a}$. When using the Geroch-Held-Penrose formalism, I will write primed variables, such as $\kappa^{\prime}, \sigma^{\prime}, \rho^{\prime}$ and $\tau^{\prime}$, as their Newman-Penrose equivalents $-v,-\lambda,-\mu$ and $-\pi$. 
Bianchi and Maxwell equations in the Appendix and I repeat part of the reasoning of [1]: choosing a null-rotation about $\boldsymbol{k}$ such that $\Phi_{1}=0$, it follows that $\Phi_{2} \neq 0 \S$. Using the GHP formalism the Maxwell equations $(64,65)$ and Bianchi equations (66-69) become then

$$
\begin{aligned}
& \text { ð } \Phi_{0}=0, \\
& \mathrm{\partial}^{\prime} \Phi_{0}=-\pi \Phi_{0}, \\
& \mathrm{P} \Phi_{0}=0, \\
& \mathrm{P}^{\prime} \Phi_{0}=-\mu \Phi_{0}, \\
& \partial \Phi_{2}=-v \Phi_{0}+\tau \Phi_{2}, \\
& \mathrm{P} \Phi_{2}=-\lambda \Phi_{0}+\rho \Phi_{2}, \\
& \partial \Psi_{2}=-\pi \Phi_{0} \overline{\Phi_{2}}+3 \tau \Psi_{2}, \\
& \mathrm{P} \Psi_{2}=\mu\left|\Phi_{0}\right|^{2}+3 \rho \Psi_{2},
\end{aligned}
$$

after which the commutators $\left[\varlimsup^{\prime}, ð\right],\left[ð^{\prime}, \mathbf{P}\right],\left[ð, \mathbf{P}^{\prime}\right]$ and $\left[\mathbf{P}^{\prime}, \mathbf{P}\right]$ applied to $\Phi_{0}$ give

$$
\begin{aligned}
\mathrm{\partial} \pi & =(3 \rho-\bar{\rho}) \mu-2 \Psi_{2}+\frac{R}{12}, \\
\mathrm{P} \pi & =3 \rho \pi, \\
\mathrm{\partial} \mu & =\bar{\lambda} \pi+3 \mu \tau, \\
\mathrm{P} \mu & =\pi(\bar{\pi}+3 \tau)+2 \Psi_{2}-\frac{R}{12} .
\end{aligned}
$$

Herewith GHP equation (63') becomes a simple algebraic equation for $\Psi_{2}$,

$$
\Psi_{2}=\rho \mu-\tau \pi+\frac{R}{12},
$$

the $\mathrm{P}$ derivative of which, using $(11,13,57,59)$, results in $\rho R=0$.

As $\rho=0$ would imply $\Phi_{0}=0$, it follows that an algebraically special Einstein-Maxwell solution possessing a shear-free and geodesic multiple Debever-Penrose vector, which is not a PND of $\boldsymbol{F}$, necessarily has a vanishing cosmological constant. The corresponding class of solutions is non-empty, as it contains the Griffiths metrics[2], encompassing as special cases the metrics of $[7,8,9,10]$.

In [2] Griffiths claimed that for $\pi=0$ both null-rays $k$ and $\ell$ are necessarily non-twisting $(\bar{\rho}-\rho=\bar{\mu}-\mu=0)$. As a consequence it was also claimed in [1] that the Griffiths metrics are uniquely characterised by the condition $\pi=0$. However, when $\pi=0$ the only conclusion to be drawn from $(10,14)$ is that $\mu(\bar{\rho}-\rho)=0$. When $\rho$ is real this indeed leads to the metrics of [2], but the case $\mu=0$ appears to have been overlooked and leads, as shown in the section below, to new classes of solutions $\|$.

$\S$ with $\Phi_{2}=0 \ell$ becomes geodesic and shear-free and the Goldberg-Sachs theorem implies $\Psi_{3}=\Psi_{4}=0$. The Petrov type would then be D, in which case $[4,5]$ the only null Einstein-Maxwell solutions are given by the (doubly aligned) Robinson-Trautman metrics.

$\|$ The case $\mu=0$ is not to be regarded as a Kundt family, as the null ray generated by $\ell$ is neither geodesic nor shear-free. 


\section{The twisting and non-expanding family}

When $\pi=0$ and $\mu=0$ the equations of the previous paragraph immediately imply $\Psi_{0}=$ $\Psi_{1}=\Psi_{2}=0$ and $\Psi_{3}=\rho v-\lambda \tau$. As little progress appears to be possible in the general case, I restrict to solutions for which $k$ is non-expanding $(\rho+\bar{\rho}=0)$. Acting on this condition with the $\partial$ and $\mathbf{P}$ operators, the GHP equations yield $\tau=0$ and

$$
\rho^{2}+\left|\Phi_{0}\right|^{2}=0,
$$

the $\partial$ derivative of which implies $\lambda=\Phi_{2} \overline{\Phi_{0}} \rho^{-1}$. Translating these results into NewmanPenrose language and fixing a boost and spatial rotation in the $\boldsymbol{k}, \boldsymbol{\ell}$ and $\boldsymbol{m}, \overline{\boldsymbol{m}}$ planes such that $\Phi_{0}=1$ and $\rho=i$, it follows that the only non- 0 spin coefficients are $\rho, v$ and $\lambda=-i \Phi_{2}$, with the only non-vanishing components of the Weyl spinor being $\Psi_{3}=i v$ and $\Psi_{4}$. As $[D, \Delta]=0$ coordinates $u, v$ and $\zeta, \bar{\zeta}$ exist such that $D=\partial_{u}, \Delta=\partial_{v}$ and

$$
\delta=e^{-i u}\left(\xi \partial_{\zeta}+\eta \partial_{\bar{\zeta}}+P \partial_{u}+Q \partial_{v}\right)
$$

$\xi, \eta, P, Q$ being arbitrary functions. The $e^{-i u}$ factor is included for convenience: applying the $[\delta, D]$ commutator to $u, v$ and $\zeta$ shows that $\xi, \eta, P, Q$ are functions of $v, \zeta, \bar{\zeta}$ only. Introducing new variables $n=e^{-i u} v$ and $\phi=e^{-2 i u} \Phi_{2}$ it follows that also $n$ and $\phi$ depend on $v, \zeta, \bar{\zeta}$ only, with the full set of Jacobi and field equations reducing to the following system of pde's:

$$
\begin{aligned}
& P_{v}+i \bar{P} \bar{\phi}-\bar{n}=0, \\
& Q_{v}+i \bar{Q} \bar{\phi}=0, \\
& \xi_{v}+i \bar{\eta} \bar{\phi}=0, \\
& \eta_{v}+i \bar{\xi} \bar{\phi}=0, \\
& e^{-i u} \bar{\delta} P-e^{i u} \delta \bar{P}-2 i|P|^{2}=0, \\
& e^{-i u} \bar{\delta} Q-e^{i u} \delta \bar{Q}-2 i \Re(Q \bar{P}-1)=0, \\
& e^{-i u} \bar{\delta} \xi-e^{i u} \delta \bar{\eta}-i(\xi \bar{P}+\bar{\eta} P)=0, \\
& e^{i u} \delta n=-i P n+2|\phi|^{2}, \\
& e^{i u} \delta \phi=-2 i P \phi-n,
\end{aligned}
$$

with the $\Psi_{4}$ component of the Weyl spinor given by $\Psi_{4}=i e^{2 i u}(\bar{n} \bar{P}+\Delta \phi)+e^{i u} \bar{\delta} n$.

\section{The case $\phi=\phi(\zeta, \bar{\zeta})$}

Assuming $\phi=\phi(\zeta, \bar{\zeta})$, writing $\phi=H^{2} h^{2}$ with $H>0$ and $|h|=1,(18,19,20)$ integrate to $Q=$ $q_{1} e^{H^{2} v}+q_{2} e^{-H^{2} v}, \xi=\overline{c_{1}} e^{H^{2} v}+\overline{c_{2}} e^{-H^{2} v}, \eta=i h^{-2}\left(-c_{1} e^{H^{2} v}+c_{2} e^{-H^{2} v}\right)$ with $q_{A}, c_{A}$ depending on $\zeta, \bar{\zeta}$ only and $q_{J}+i h^{-2} \overline{q_{J}}=0(J=1,2)$. A coordinate transformation $\zeta \rightarrow \tilde{\zeta}(\zeta, \bar{\zeta})$ allows one then to put (writing $\tilde{\zeta}=x+i y$ and re-defining $q_{J}$ ),

$\delta=e^{-i u}\left[P \partial_{u}+e^{H^{2} v-C_{1}-i \frac{\pi}{4}} h^{-1}\left(\partial_{x}+q_{1} \partial_{v}\right)+e^{-H^{2} v-C_{2}+i \frac{\pi}{4}} h^{-1}\left(\partial_{y}+q_{2} \partial_{v}\right)\right]$,

with $C_{J}$ and $q_{J}$ real functions of $x$ and $y$. 
A new class of non-aligned Einstein-Maxwell solutionswith a geodesic, shearfree and non-expanding multiple Deb

An expression for $P$ is obtained from (23),

$$
\begin{aligned}
P=e^{i \frac{\pi}{4}} h^{-1}\left[e^{H^{2} v-C_{1}}\left(\frac{h_{, x}}{h}-C_{2, x}-v\left(H^{2}\right)_{, x}-q_{1} H^{2}\right)\right. \\
\left.+i e^{-H^{2} v-C_{2}}\left(\frac{h_{, y}}{h}-C_{1, y}+v\left(H^{2}\right)_{, y}+q_{2} H^{2}\right)\right],
\end{aligned}
$$

after which $n$ follows from (17),

$$
\begin{aligned}
n=-2 h H^{2}\left\{e^{H^{2} v-C_{1}-i \frac{\pi}{4}}\left[\left(1+2 v H^{2}\right) \frac{H_{, x}}{H}+C_{2, x}+q_{1} H^{2}\right]\right. \\
\left.+e^{-H^{2} v-C_{2}+i \frac{\pi}{4}}\left[\left(1-2 v H^{2}\right) \frac{H_{, y}}{H}+C_{1, y}-q_{2} H^{2}\right]\right\} .
\end{aligned}
$$

Herewith (21) becomes a polynomial identity in powers of $v$ and $e^{H^{2} v}$,

$$
v^{2} e^{2 H^{2} v} H_{, x}^{2}-v^{2} e^{-2 H^{2} v} H_{, y}^{2}+\ldots=0,
$$

showing that $H$ is necessarily constant.

Introducing new variables $r_{1}=-H^{2} q_{1}-C_{2, x}, r_{2}=H^{2} q_{2}-C_{1, y}$ the remaining coefficients of (29) lead to the equations,

$$
\begin{aligned}
& r_{1, x}-2 r_{1}^{2}-r_{1}\left(C_{1}+C_{2}\right)_{, x}=0, \\
& r_{2, y}-2 r_{2}^{2}-r_{2}\left(C_{1}+C_{2}\right)_{, y}=0 .
\end{aligned}
$$

Substituting this in (28), equation (24) becomes a Liouville equation determining $C_{1}+C_{2}$,

$$
\left(C_{1}+C_{2}\right)_{, x y}+2 H^{2} e^{C_{1}+C_{2}}=0,
$$

while (25) reduces to an identity. A final equation is (22), which now becomes

$$
r_{2, x}+r_{1, y}-H^{2} e^{C_{1}+C_{2}}=0 .
$$

The general solution of the Liouville equation being given by

$$
e^{C_{1}+C_{2}}=-\frac{a_{, x} b_{, y}}{H^{2}(a+b)^{2}},
$$

( $a=a(x)$ and $b=b(y)$ arbitrary functions), $r_{1}$ and $r_{2}$ are given by $(30,31)$ as,

$$
r_{1}=\frac{b_{, y}}{2(a+b)(1+A(a+b))}, r_{2}=\frac{a_{, x}}{2(a+b)(1+B(a+b))},
$$

with arbitrary functions $A=A(x), B=B(y)$. Herewith (33) reduces to the condition

$$
\left(\frac{\mathrm{d} A}{\mathrm{~d} a}-A^{2}\right)(1+B(a+b))^{2}+\left(\frac{\mathrm{d} B}{\mathrm{~d} b}-B^{2}\right)(1+A(a+b))^{2}=0,
$$

implying either $A_{, a}-A^{2}=B_{, b}-B^{2}=0$, or $\log \frac{1+B(a+b)}{1+A(a+b)}$ being separable in $x$ and $y$. As the latter condition again can be shown to imply $A_{, a}-A^{2}=B_{, b}-B^{2}=0$, we conclude that the general solution is given by

$$
r_{1}=\frac{a_{, x}}{2(a+b)} \frac{k-k_{0} b}{k+k_{0} a}, r_{2}=\frac{b_{, y}}{2(a+b)} \frac{k-k_{0} a}{k+k_{0} b},
$$

with either $k_{0}=1$ and $k$ an arbitrary (real) constantII, or $k_{0}=0, k=1$ (corresponding to the special case $A=B=0$ ).

II which can be taken to be 0 or 1 
The resulting metric appears to contain two arbitrary functions, being the phase factor $h(x, y)$ of $\phi$ and the function $F(x, y)$ defined by $e^{C_{1}-C_{2}}=-\frac{a_{,} x}{b_{, y}} e^{2 F}$. These however can be eliminated by the coordinate transformations $u \rightarrow u-i \log h$ and $v \rightarrow H^{2} v-F$, after which the dual basis takes the form,

$$
\begin{aligned}
\boldsymbol{\omega}^{1} & =\frac{e^{i u}}{2 H(a+b)}\left(e^{i \frac{\pi}{4}-v} \mathrm{~d} a-e^{-i \frac{\pi}{4}+v} \mathrm{~d} b\right), \\
\boldsymbol{\omega}^{3} & =\frac{1}{H^{2}} \mathrm{~d} v-\frac{1}{H^{2}(a+b)}\left[\frac{(2 a+b) k_{0}+k}{2\left(k_{0} a+k\right)} \mathrm{d} a-\frac{(a+2 b) k_{0}+k}{2\left(k_{0} b+k\right)} \mathrm{d} b\right], \\
\boldsymbol{\omega}^{4} & =\mathrm{d} u+\frac{1}{2(a+b)}\left[\frac{k_{0} a-k}{k_{0} b+k} e^{-2 v} \mathrm{~d} a-\frac{k_{0} b-k}{k_{0} a+k} e^{2 v} \mathrm{~d} b\right],
\end{aligned}
$$

$\left(k_{0}, k\right)=(1,0),(1,1)$ or $(0,1)$.

\section{Discussion}

The null tetrad (38-40) determines a (presumably) new family of Einstein-Maxwell solutions with zero cosmological constant and with Maxwell field and energy-momentum tensor given by

$$
\begin{aligned}
& \boldsymbol{F}=i H^{2}\left(\boldsymbol{\omega}^{1}-\boldsymbol{\omega}^{2}\right) \wedge \boldsymbol{\omega}^{3}+i\left(e^{-2 i u} \boldsymbol{\omega}^{1}-e^{2 i u} \boldsymbol{\omega}^{2}\right) \wedge \boldsymbol{\omega}^{4}, \\
& \boldsymbol{T}=2 H^{2}\left(e^{-2 i u} \boldsymbol{\omega}^{1} \otimes \boldsymbol{\omega}^{1}+e^{2 i u} \boldsymbol{\omega}^{2} \otimes \boldsymbol{\omega}^{2}+H^{2} \boldsymbol{\omega}^{3} \otimes \boldsymbol{\omega}^{3}\right)+2 \boldsymbol{\omega}^{4} \otimes \boldsymbol{\omega}^{4} .
\end{aligned}
$$

The Petrov type is III, with the multiple Debever-Penrose vector $\boldsymbol{k}=\partial_{u}$ being geodesic, shear-free and twisting but non-expanding. The real null vector $\ell$, fixed by a null-rotation such that $\Phi_{1}=0$, is non-diverging, but is non-geodesic and has non-vanishing shear. It follows that figure (2) in [1] has to be amended as in Fig. 1 below.

For all solutions $\partial_{u}$ is clearly a null Killing vector. While in general (i.e. with $k$ and $k_{0} \neq 0$ ) the isometry group is 2-dimensional, with the second Killing vector given by

$$
\boldsymbol{K}_{2}=k_{0}^{2}(a+b) \partial_{v}+\left(a^{2} k_{0}^{2}-k^{2}\right) \partial_{a}-\left(b^{2} k_{0}^{2}-k^{2}\right) \partial_{b},
$$

the special cases $k_{0}=0, k=1$ and $k_{0}=1, k=0$ admit a 3-dimensional group of isometries, with third Killing vector

$$
\boldsymbol{K}_{3}=a \partial_{a}+b \partial_{b}
$$

In the latter cases the isometry group has Bianchi type III, with the orbits being time-like hypersurfaces parametrized by the null coordinate $v$. For the case $k_{0}=0, k=1$ the tetrad simplifies to

$$
\begin{aligned}
\boldsymbol{\omega}^{1} & =\frac{e^{i u}}{2 H(a+b)}\left[e^{i \frac{\pi}{4}-v} \mathrm{~d} a-e^{-i \frac{\pi}{4}+v} \mathrm{~d} b\right], \\
\boldsymbol{\omega}^{3} & =\frac{1}{H^{2}}\left[\mathrm{~d} v-\frac{1}{2(a+b)} \mathrm{d}(a-b)\right], \\
\omega^{4} & =\mathrm{d} u-\frac{1}{2(a+b)}\left[e^{-2 v} \mathrm{~d} a-e^{2 v} \mathrm{~d} b\right]
\end{aligned}
$$




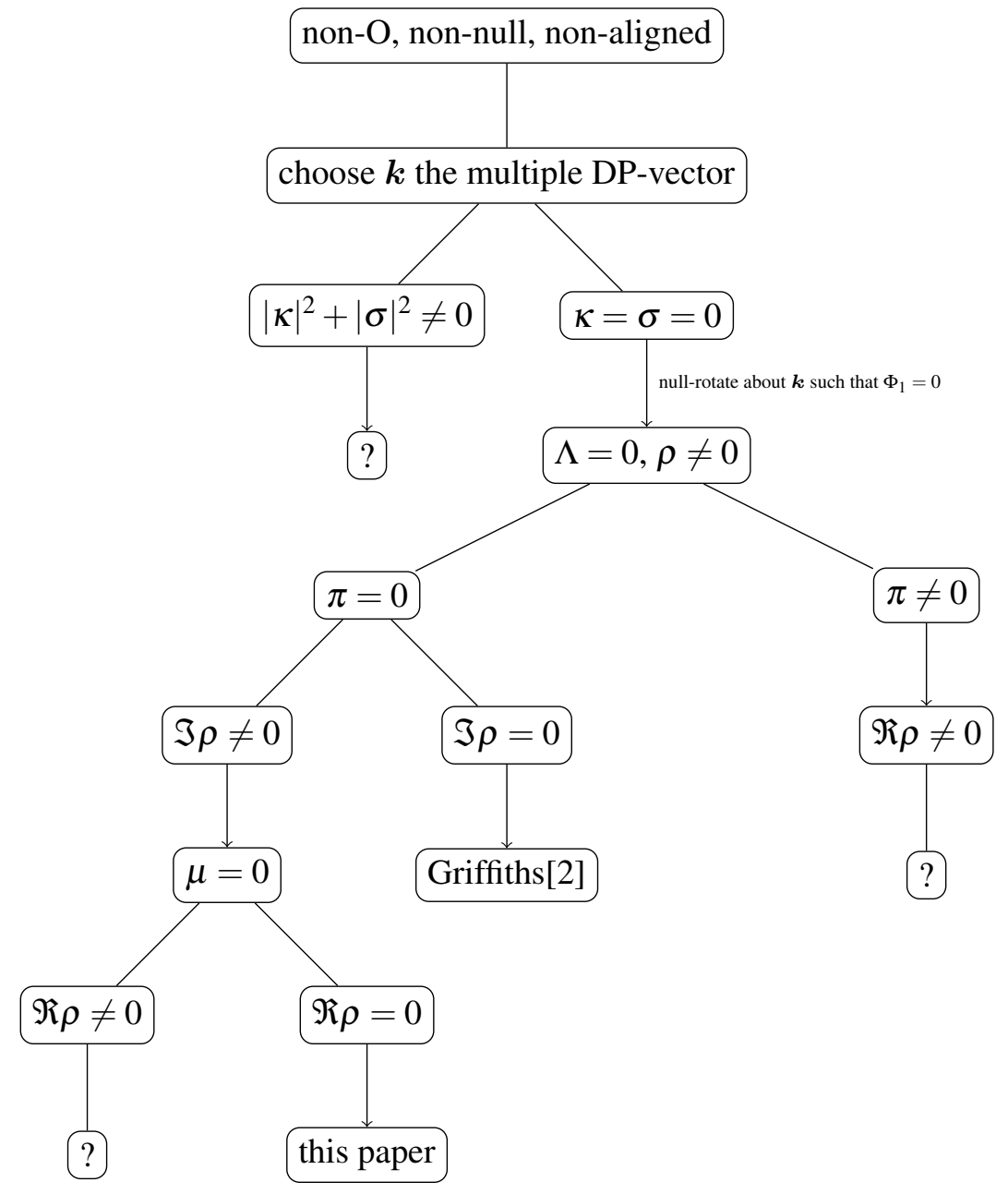

Figure 1. Algebraically special non-nul Einstein-Maxwell solutions for which the multiple Weyl-PND $\boldsymbol{k}$ is not a PND of $\boldsymbol{F}$.

and the line-element becomes

$\mathrm{d} s^{2}=\frac{1}{H^{2}}\left[-2 \mathrm{~d} v+\frac{1}{a+b} \mathrm{~d}(a-b)\right] \mathrm{d} u+\frac{1}{H^{2}(a+b)}\left(e^{-2 v} \mathrm{~d} a-e^{2 v} \mathrm{~d} b\right) \mathrm{d} v+\frac{\cosh 2 v}{H^{2}(a+b)^{2}} \mathrm{~d} a \mathrm{~d} b$.

The non-0 components of the Weyl-spinor are then given by

$$
\begin{aligned}
& \Psi_{3}=-H^{3} e^{i\left(u-\frac{\pi}{4}\right)}\left(e^{v}+i e^{-v}\right) \\
& \Psi_{4}=2 H^{4} e^{2 i u} \cosh 2 v .
\end{aligned}
$$

All the Carminati-McLenaghan invariants are regular functions of the essential coordinate $v$ over the interval $]-\infty,+\infty\left[\right.$. The same holds for the case $k_{0}=1, k=0$ (in which the essential coordinate is $v+\log (b / a))$, but although all the (CM-) invariants are transformed into each other under the coordinate transformation $v \rightarrow v+\log (b / a)$ the two special cases are inequivalent.

$\partial_{u}$ being Killing vector, it might look peculiar that the Weyl spinor components $\Psi_{3}$ and $\Psi_{4}$ still depend on $u$, even though the frame was "invariantly" fixed. This is due to the fixation having been done by means of a null rotation putting $\Phi_{0}=1$ : the resulting frame scalars are 
A new class of non-aligned Einstein-Maxwell solutionswith a geodesic, shearfree and non-expanding multiple Deb

then not genuine Cartan invariants and, as the Maxwell field itself does not inherit the spacetime symmetries ( $\boldsymbol{F}$ is not Lie-propagated along the integral curves of the null Killing vector $\partial_{u}$ ), the frame scalars depend on $u$ as well. This remark also shows that the present solutions are distinct from the Lucács et al. solutions admitting null-Killing vectors [11], as there the Maxwell field does inherit all the space-time symmetries.

\section{Appendix A}

Weights $^{+}$of the spin-coefficients, the Maxwell and Weyl spinor components and the GHP operators:

$$
\begin{aligned}
& \kappa:[3,1], v:[-3,-1], \sigma:[3,-1], \lambda:[-3,1], \\
& \rho:[1,1], \mu:[-1,-1], \tau:[1,-1], \pi:[-1,1], \\
& \Phi_{0}:[2,0], \Phi_{1}:[0,0], \Phi_{2}:[-2,0], \\
& \Psi_{0}:[4,0], \Psi_{1}:[2,0], \Psi_{2}:[0,0], \Psi_{3}:[-2,0], \Psi_{4}:[-4,0], \\
& \partial:[1,-1], \partial^{\prime}:[-1,1], \mathrm{P}^{\prime}:[-1,-1], \mathrm{P}:[1,1] .
\end{aligned}
$$

The prime operation is an involution with

$$
\begin{aligned}
& \kappa^{\prime}=-v, \sigma^{\prime}=-\lambda, \rho^{\prime}=-\mu, \tau^{\prime}=-\pi, \\
& \Psi_{0}{ }^{\prime}=\Psi_{4}, \Psi_{1}{ }^{\prime}=\Psi_{3}, \Psi_{2}{ }^{\prime}=\Psi_{2}, \\
& \Phi_{0}^{\prime}=-\Phi_{2}, \Phi_{1}^{\prime}=-\Phi_{1} .
\end{aligned}
$$

The GHP commutators acting on $(p, q)$ weighted quantities are given by:

$$
\begin{aligned}
& {\left[\mathbf{P}, \mathbf{P}^{\prime}\right]=(\pi+\bar{\tau}) ð+(\bar{\pi}+\tau) \widetilde{\partial}^{\prime}+\left(\kappa v-\pi \tau+\frac{R}{24}-\Phi_{11}-\Psi_{2}\right) p} \\
& +\left(\overline{\kappa \nu}-\overline{\pi \tau}+\frac{R}{24}-\Phi_{11}-\bar{P}_{2}\right) q, \\
& {\left[ð, \check{\partial}^{\prime}\right]=(\mu-\bar{\mu}) \mathrm{P}+(\rho-\bar{\rho}) \mathrm{P}^{\prime}+\left(\lambda \sigma-\mu \rho-\frac{R}{24}-\Phi_{11}+\Psi_{2}\right) p} \\
& -\left(\overline{\lambda \sigma}-\overline{\mu \rho}-\frac{R}{24}-\Phi_{11}+\bar{P}_{2}\right) q, \\
& {[\mathrm{P}, ð]=\bar{\pi} \mathrm{P}-\kappa \mathrm{P}^{\prime}+\bar{\rho} \partial+\sigma \check{\partial}^{\prime}+\left(\kappa \mu-\sigma \pi-\Psi_{1}\right) p} \\
& +\left(\overline{\kappa \lambda}-\overline{\pi \rho}-\Phi_{01}\right) q .
\end{aligned}
$$

GHP equations:

$$
\begin{aligned}
& \mathrm{P} \rho-\partial^{\prime} \kappa=\rho^{2}+\sigma \bar{\sigma}-\bar{\kappa} \tau+\kappa \pi+\Phi_{00}, \\
& \mathrm{P} \sigma-ð \kappa=(\rho+\bar{\rho}) \sigma+(\bar{\pi}-\tau) \kappa+\Psi_{0}, \\
& \mathrm{P} \tau-\mathrm{P}^{\prime} \kappa=(\tau+\bar{\pi}) \rho+(\bar{\tau}+\pi) \sigma+\Phi_{01}+\Psi_{1}, \\
& \mathrm{P} v-\mathrm{D}^{\prime} \pi=(\pi+\bar{\tau}) \mu+(\bar{\pi}+\tau) \lambda+\Psi_{3}+\Phi_{1} \Phi_{2}, \\
& \text { } \rho-\partial^{\prime} \sigma=(\rho-\bar{\rho}) \tau+(\mu-\bar{\mu}) \kappa+\Phi_{01}-\Psi_{1},
\end{aligned}
$$

+ Objects $x$ transforming under boosts and rotations as $x \rightarrow A^{\frac{p+q}{2}} e^{i \frac{p-q}{2}} x$ are called well-weighted of type $(p, q)$. 
A new class of non-aligned Einstein-Maxwell solutionswith a geodesic, shearfree and non-expanding multiple Deb

$$
\begin{aligned}
& \mathrm{P}^{\prime} \sigma-ð \tau=-\sigma \mu-\bar{\lambda} \rho-\tau^{2}+\kappa \bar{v}-\Phi_{02}, \\
& \mathrm{P}^{\prime} \rho-\check{\partial}^{\prime} \tau=-\bar{\mu} \rho-\lambda \sigma-\tau \bar{\tau}+\kappa \nu-\frac{R}{12}-\Psi_{2} .
\end{aligned}
$$

Maxwell equations:

$$
\begin{aligned}
& \mathrm{P} \Phi_{1}-\check{\partial}^{\prime} \Phi_{0}=\pi \Phi_{0}+2 \rho \Phi_{1}-\kappa \Phi_{2}, \\
& \mathrm{P} \Phi_{2}-\varpi^{\prime} \Phi_{1}=-\lambda \Phi_{0}+2 \pi \Phi_{1}+\rho \Phi_{2} .
\end{aligned}
$$

Bianchi equations (with $\Phi_{I J}=\Phi_{I} \overline{\Phi_{J}}$ and $\Lambda=R / 4=$ constant):

$$
\begin{aligned}
& \bar{\partial}^{\prime} \Psi_{0}-\mathrm{P} \Psi_{1}+\mathrm{P} \Phi_{01}-\partial \Phi_{00}=-\pi \Psi_{0}-4 \rho \Psi_{1}+3 \kappa \Psi_{2}+\bar{\pi} \Phi_{00}+2 \bar{\rho} \Phi_{01}+2 \sigma \Phi_{10} \\
& -2 \kappa \Phi_{11}-\bar{\kappa} \Phi_{02}, \\
& \mathrm{P}^{\prime} \Psi_{0}-ð \Psi_{1}+\mathrm{P} \Phi_{02}-ð \Phi_{01}=-\mu \Psi_{0}-4 \tau \Psi_{1}+3 \sigma \Psi_{2}-\bar{\lambda} \Phi_{00}+2 \bar{\pi} \Phi_{01}+2 \sigma \Phi_{11} \\
& +\bar{\rho} \Phi_{02}-2 \kappa \Phi_{12} \\
& 3 \check{\partial}^{\prime} \Psi_{1}-3 \mathrm{P} \Psi_{2}+2 \mathrm{P} \Phi_{11}-2 \partial \Phi_{10}+\check{\partial}^{\prime} \Phi_{01}-\mathrm{P}^{\prime} \Phi_{00}=3 \lambda \Psi_{0}-9 \rho \Psi_{2}-6 \pi \Psi_{1}+6 \kappa \Psi_{3} \\
& +(\bar{\mu}-2 \mu) \Phi_{00}+2(\pi+\bar{\tau}) \Phi_{01}+2(\tau+\bar{\pi}) \Phi_{10}+2(2 \bar{\rho}-\rho) \Phi_{11} \\
& +2 \sigma \Phi_{20}-\bar{\sigma} \Phi_{02}-2 \bar{\kappa} \Phi_{12}-2 \kappa \Phi_{21}, \\
& 3 \mathrm{P}^{\prime} \Psi_{1}-3 \partial \Psi_{2}+2 \mathrm{P} \Phi_{12}-2 \partial \Phi_{11}+\check{\partial}^{\prime} \Phi_{02}-\mathrm{P}^{\prime} \Phi_{01}=3 v \Psi_{0}-6 \mu \Psi_{1}-9 \tau \Psi_{2}+6 \sigma \Psi_{3} \\
& -\bar{v} \Phi_{00}+2(\bar{\mu}-\mu) \Phi_{01}-2 \bar{\lambda} \Phi_{10}+2(\tau+2 \bar{\pi}) \Phi_{11}+(2 \pi+\bar{\tau}) \Phi_{02} \\
& +2(\bar{\rho}-\rho) \Phi_{12}+2 \sigma \Phi_{21}-2 \kappa \Phi_{22} \text {. }
\end{aligned}
$$

\section{Acknowledgment}

All calculations were done using the Maple symbolic algebra system, while the properties of the metric (48) were checked with the aid of Maple's DifferentialGeometry package[12].

[1] Van den Bergh N 2017 Gen. Rel. Grav. 49, 1

[2] Griffiths J B 1986 Gen. Rel. Grav. 18, 4

[3] Goldberg J N and Sachs R K 1962 Acta Phys. Pol. Suppl. 22, 13

[4] Debever R, Van den Bergh N and Leroy J 1989 Class. Quantum Grav. 6, 1373

[5] Van den Bergh N 1989 Class. Quantum Grav. 6, 1871

[6] Kramer D, Stephani H, MacCallum M A H, Hoenselaers C and Herlt E, Exact solutions of Einstein's field equations, Cambridge University Press, 2003

[7] Cahen M and Leroy J 1966 J. Math. Mech. 16, 501

[8] Cahen M and Spelkens J 1967 Bull. Soc. Math. Belg. 53, 817

[9] Griffiths J B 1983 J. Phys. A 16, 1175

[10] Szekeres P 1966 J. Math. Phys. 7, 751

[11] Lucács B, Perjés Z and Sebestyén Á 1981 J. Math. Phys. 22, 1248

[12] Anderson I M and Torre C G, 2012 J. Math. Phys. 53, 013511 